\title{
Analyst
}

\section{Investigating cellular responses to novel chemotherapeutics in renal cell carcinoma using SR-FTIR spectroscopy $\dagger$}

\author{
C. Hughes, ${ }^{a b}$ M. D. Brown, ${ }^{b}$ N. W. Clarke, ${ }^{b}$ K. R. Flower ${ }^{c}$ and P. Gardner ${ }^{\star a}$ \\ Received 14th May 2012, Accepted 8th August 2012 \\ DOI: 10.1039/c2an35632e
}

\begin{abstract}
SR-FTIR spectroscopy was evaluated as a technique to discriminate spectral signals of cellular response at the single cell level, when cancer cells are exposed to chemotherapeutics. 5-Fluorouracil, an established drug of known mode of action, was tested against a renal carcinoma cell line (Caki-2), along with two experimental analogues of gold-based compounds. The use of unsupervised principal component analysis (PCA) failed to clearly define any distinction between control and drug treated cell spectra. Supervised principal component linear discriminant analysis (PC-LDA) did have some potential to reveal signatures of cell response and repair but again failed to distinctly discriminate groups of spectra with different drug treatments. Alternatively, clear PCA discrimination was observed in spectra from average cell populations via single point benchtop spectroscopy, probing several cells simultaneously with an increased aperture. The Caki-2 cell line initially appeared to be sensitive to the novel compounds, inducing a cellular response prior to subsequential cell recovery which was assessed by both PCA and cell viability assays.
\end{abstract}

\section{Introduction}

Cells are said to have specific infrared spectroscopic signatures in terms of cell cycle, viability, apoptosis and stress. ${ }^{1-7}$ The discovery of a spectroscopic signature, unique to a particular mode of drug action, would be highly beneficial for the early stages of the drug development process, as novel chemotherapeutic agents with potential regularly fail at various stages during their progression and through to clinical trials. Currently, high throughput procedures used in the development of novel compounds include cytotoxicity multi-cell line panel testing and the assessment of agent interaction and involvement in parts of numerous biological pathways. ${ }^{8}$

Implementing FTIR spectroscopy as a tool in this area would only be appropriate for the assessment of global changes in cellular chemistry. Attempting to understand the drug activity upon cellular processes is a complex area. Despite this fact, distinct biomarkers for drug modality may be discovered, using chemometric techniques to discriminate drugs that invoke different mechanisms or have different modes of action. The

${ }^{a}$ Manchester Interdisciplinary Biocentre, University of Manchester, 131 Princess Street, Manchester, UK M1 7DN. E-mail: peter.gardner@ manchester.ac.uk

${ }^{b}$ Genito-Urinary Cancer Research Group, School of Cancer and Enabling Sciences, Paterson Institute for Cancer Research, University of Manchester, The Christie NHS Foundation Trust, Manchester Academic Health Sciences Centre, Manchester, UK M20 4BX

'School of Chemistry, University of Manchester, Manchester, M13 9PL, $U K$

$\dagger$ Electronic supplementary information (ESI) available. See DOI: $10.1039 / \mathrm{c} 2 \mathrm{an} 35632 \mathrm{e}$ early detection of cellular responses to drugs in the infrared could highlight differences that may not be achievable by conventional testing. Common biomarkers may be deduced from drug classes that exhibit similar cellular responses, helping to predict and understand drug efficacy.

Renal cell carcinoma is one of the most aggressive and challenging cancers to treat with chemotherapy, therefore the discovery of novel compounds with high efficacy in this field would be extremely welcomed. In this current study the renal carcinoma cell line, Caki-2 has been dosed with a drug of known mode of action as well as a set of novel gold-based compounds with unknown efficacy and evaluated at different time points. The novel compounds studied were gold analogues, KF0101 and KF0113 (referred to as KF1 and KF13 in future). ${ }^{9}$ The compounds exhibit some efficacy in a number of cancers through testing in the National Cancer Institutes' (NCI) 60 panel. This is a collection of 60 human cancerous cell lines commonly used as a key tool to develop and screen anticancer drugs. ${ }^{10}$

\section{Methods and materials}

\section{Cell culture}

The commercially available Caki- 2 cell line, originally established from a primary clear cell carcinoma of the kidney from a 69 year old male caucasian, was cultured in Dulbecco's Modified Eagle Medium (DMEM) with foetal calf serum (FCS) (15\%) and L-glutamine $(1 \%)$ at $37{ }^{\circ} \mathrm{C}$ in a humidified atmosphere $\left(5 \% \mathrm{CO}_{2}\right)$ and maintained at a $70 \%$ confluence rate until required for experimentation. 


\section{Determination of inhibitory concentrations}

An inhibitory concentration (IC) value for the anticancer agents was determined by the sulforhodamine B (SRB) assay as described by Vichai and Kirtikara. ${ }^{11}$ Cells were seeded at a predefined density (3000 cells in $100 \mu \mathrm{L}$ medium per well) in triplicate and left for 24 hours to ensure cells were well-established and adhered to the bottom of the well plate. A range of 12 drug concentrations between 0.15 and $20.0 \mu \mathrm{M}$ were used by the addition of drug/media $(100 \mu \mathrm{L}$ at twice the desired concentration) to the established cells in media $(100 \mu \mathrm{L})$ giving the correct final concentration range. For the control group, media only was added $(100 \mu \mathrm{L}$ ). (A vehicle of DMSO diluted to the strongest working concentration in the assay was also performed however no significant difference was observed.) Well plates were incubated at $37^{\circ} \mathrm{C}$ for 3 days in $5 \% \mathrm{CO}_{2}$ in air. After 3 days the well solution was discarded and wells washed with PBS $(\times 2100 \mu \mathrm{L})$. The remaining cells were fixed with trichloroacetic acid (TCA) $\left(100 \mu \mathrm{L} ; 10 \% \mathrm{w} / \mathrm{v} \mathrm{H}_{2} \mathrm{O}\right), 4{ }^{\circ} \mathrm{C}$ for $1 \mathrm{~h}$. The wells were washed with PBS $(\times 2100 \mu \mathrm{L})$ and dried at room temperature (RT) for 1-2 hours. The cells were stained with SRB $\left(100 \mu \mathrm{L} ; 0.4 \% \mathrm{w} / \mathrm{v} \mathrm{H}_{2} \mathrm{O}\right)$ and left for 15 minutes at RT. The plates were then washed with acetic acid $\left(1 \% \mathrm{w} / \mathrm{v} \mathrm{H}_{2} \mathrm{O}\right)$ until the acid ran clear and dried at RT for 1-2 hours. The protein residues were then re-suspended with Tris- $\mathrm{HCl}(100 \mu \mathrm{L} ; 1.5 \mathrm{M}$; $\mathrm{pH} 8.8$ ) with agitation on a shaker for 5 minutes before reading on a Versamax plate reader (Molecular Devices) at $490 \mathrm{~nm}$ absorbance.

Three independent experiments were performed, each with triplicate wells per concentration point. The mean absorbance values were then calculated and exported to Graphpad Prism for normalisation and plotted in the logarithmic form to determine the $\mathrm{IC}_{50}$ values for each agent \pm standard error (Fig. 1). The concentrations were calculated as; KF0101 [3.51 $\mu \mathrm{M}]$; KF0113 $[3.52 \mu \mathrm{M}]$; 5FU $[2.39 \mu \mathrm{M}]$. Concentration ranges at the $95 \%$ confidence interval were $[3.165-3.902 \mu \mathrm{M}],[3.118-3.973 \mu \mathrm{M}]$ and $[2.087-2.745 \mu \mathrm{M}]$ respectively.
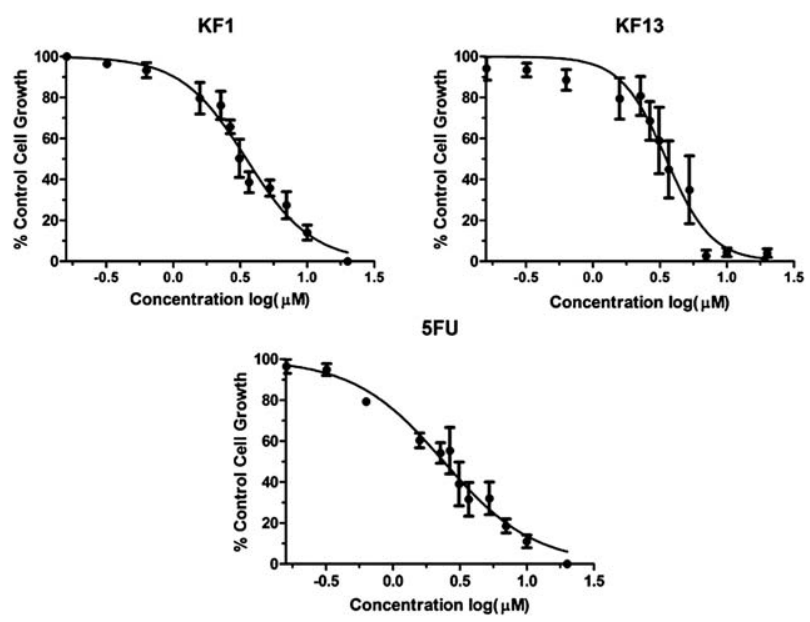

Fig. 1 Dose response curves with mean values \pm standard error from triplicate experiments to determine $\mathrm{IC}_{50}$ values for the Caki-2 cell line from the SRB assay data.

\section{Proliferation assay}

Caki- 2 cells were added to well plates ( 6 wells per plate) at a density of $1 \times 10^{5}$ cells per well and left to establish. 72 wells were setup to allow for triplicate measurements over a 6 day period. After 24 hours, agents KF1, KF13 and 5FU were introduced at the $\mathrm{IC}_{50}$ level determined by a 3 day SRB assay $(3.51,3.52$ and $2.39 \mu \mathrm{M}$ respectively). For each subsequent day (1-6 days after drug introduction) viable cells were identified using trypan blue dye and then counted using a haemocytometer. The assay performed twice to confirm the proliferative trend of each drug treatment.

\section{FTIR sample preparation}

Caki- 2 cells were added to well plates ( 6 wells per plate) at a density of $1 \times 10^{5}$ cells per well and left to establish onto sterilised MirrIR $^{\mathrm{TM}}$ substrates. Technical replicates were made however the samples were only prepared in a single experiment. After 24 hours, agents $\mathrm{KF} 1, \mathrm{KF} 13$ and $5 \mathrm{FU}$ were introduced at the $\mathrm{IC}_{50}$ level, as determined by the SRB assay to the cells that were at a subconfluent density of approximately $50 \%$. For the later single point studies, cells were also exposed to KF13 and left to culture for a further 72 hours and both KF13 and 5FU at 144 hours. At the appropriate time point the cell-cultured slides were washed twice in PBS and fixed in formalin (4\%) for 40 minutes. The slides were then dipped in distilled water to remove any residual salt. ${ }^{12}$

\section{FTIR measurements and processing}

SR-FTIR single point transflection spectra were acquired on the SMIS beamline of the French Synchrotron Facility SOLEIL (Saint-Aubin, France) using a Nicolet Continuum XL microscope equipped with an MCT detector. ${ }^{13}$ Spectra were recorded at $4 \mathrm{~cm}^{-1}$ resolution with 256 co-scans and the size of the aperture was adjusted to match the diameter of the cell such that it was fully illuminated, typically $15 \mu \mathrm{m}^{2}$.

Single point benchtop FTIR measurements were acquired at 128 co-scans using an aperture of $100 \times 100 \mu \mathrm{m}$. Single point transflection spectra were taken using a BioRad FTS 7000 equipped with a liquid nitrogen-cooled $\mathrm{MCT}$ detector and a $\mathrm{KBr}$ beam-splitter, attached to a microscope using WinIRPro Software (Varian Inc., U.S.A.). The cells were viewed on a movable $x, y$ stage using a $15 \times$ objective lens. A total of 20 spectra for each group were acquired, sampling approximately in the order of 25 cells per spectrum.

All datasets were subject to the RMieS-EMSC correction algorithm at 150 iterations. SR-FTIR single cell spectra are perturbed by severe RMieS scattering due to the similar dimension to the incident wavelength of light and therefore require a larger number of iterations relative to non-severe scattering sample types, such as tissue. ${ }^{14,15}$ A Matrigel $^{\text {TM }}$ transflection $^{-1}$ spectrum was used as the reference. The corrected spectra were transformed to the second derivative with 7 point SavitskyGolay smoothing with a polynomial order of 3 in Matlab. Analysis was performed using the fingerprint range of 900-1825 $\mathrm{cm}^{-1}$. The corrected spectra were vector normalised and meancentred before principal component analysis (PCA) and principal component linear discriminant analysis (PC-LDA) in Matlab (Mathworks Inc.). 


\section{Results and discussion}

\section{Assessment of SR-FTIR spectral signatures 24 hours after drug exposure}

PCA. Spectra of drug-treated cells after 24 hours of drug exposure were assessed against the (non-drug treated) control group (Fig. 2). There was little difference to be observed in the mean spectra for each cell type in general (Fig. 2a), although subtle absorbance differences were observed in spectral peaks associated with $\delta_{\text {as }}(\mathrm{CH})_{2}$ at $\sim 1480 \mathrm{~cm}^{-1}, \nu_{\text {as }}(\mathrm{PO})_{2}$ at $\sim 1240 \mathrm{~cm}^{-1}$ and $\nu_{\mathrm{s}}(\mathrm{PO})_{2}$ at $1080 \mathrm{~cm}^{-1}$ were noted for $\mathrm{KF} 1$ and KF13-treated cells.

No distinction could be made between control cells and drug treated cells using principal component analysis (PCA) (Fig. 2b). This may be due to the intra-sample heterogeneity observed in the cell populations. Cells exhibit specific chemistry in a certain phase of the cell cycle and therefore it may be difficult to detect any chemistry purely associated with drug response from influence of the cell cycle. This has also been suggested in parallel research. ${ }^{16}$ It is known that $5 \mathrm{FU}$ does affect cell cycle in the $\mathrm{S}$ phase; however at this time there is no evidence to suggest that the gold compounds have any cell cycle influence.

Additionally, the use of $\mathrm{IC}_{50}$ values, by their definition, will equate to the inhibition of half the population of cells at that specific concentration. The implication for single cell analysis will mean that the agent may elicit a cellular response in a given period of time for some cells, but not others.

PC-LDA. PC-LDA was assessed as to whether overall differences could be maximised in an attempt to detect general trends. However, the 'ideal' number of principal components to use in a model can be subjective, although there are a number of ways to deduce this optimal number for a given dataset. Along with the ' $(n-g) / 3$ ' over-fitting rule, ${ }^{17}$ the Kaiser rule recommends discarding principal components after the eigenvalues are no longer equal to or above $1.0{ }^{18}$ Another approach is to use the number of PCs that explain $95 \%$ of total variance. A fourth method commonly used is Cattell's scree plot analysis, whereby the number of components taken should equal the point before the plot reaches the 'elbow'. ${ }^{19}$ Finally, Monte Carlo PCA for Horn's parallel analysis can be used whereby PCA is performed to obtain eigenvalues for a correlation matrix constructed by random numbers, equal in size to the experimental data. ${ }^{20}$ The scree plots for simulated and experimental data are compared and the threshold is the PC before the experimental plot crosses over the simulated plot. ${ }^{21}$ Using these guidelines for the same datasets used in PCA, the highest suggested number of components was calculated as four for PC-LDA (ESI Fig. $1 \dagger$ ). There was however still overlap of drug-treated and control spectra (ESI Fig. $2 \dagger$ ). PC-LDA was repeated for the second derivativeequivalent of the dataset. Generally it was noted that more PCs were required to explain the same amount of variance in the $2^{\text {nd }}$ derivative dataset (Fig. 3). Derivatives assess the rate of change in absorbance with respect to wavenumber and consequently the strong amide I and II signals dominate derivative spectra and subsequent loading plots. Information from other regions of the spectrum with small derivative peaks do not become significant until later components are used. As more and more PCs are used, however, more noise contributes to the signal and a cautionary balance must be made. These signals, however, were indistinguishable from the signals in the DF2 loading plot (not pictured). Discriminant analysis (DA), like PCA is a form of eigen-analysis and for $k$ groups, DA finds the $k-1$ discriminant axes that maximally separate the $k$ groups. In a two group case of LDA,
I. a

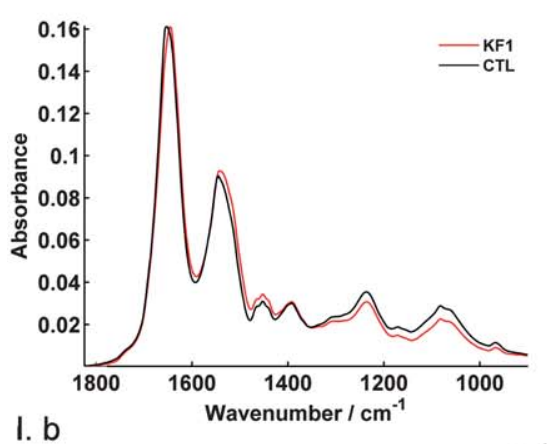

I. b

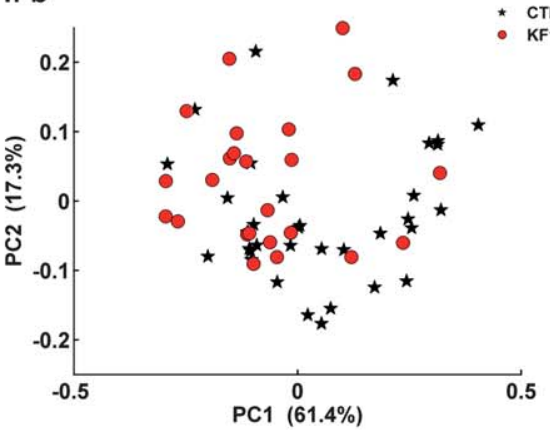

II. a

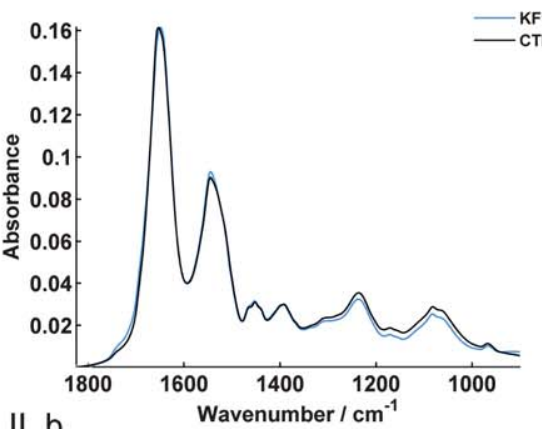

III. a
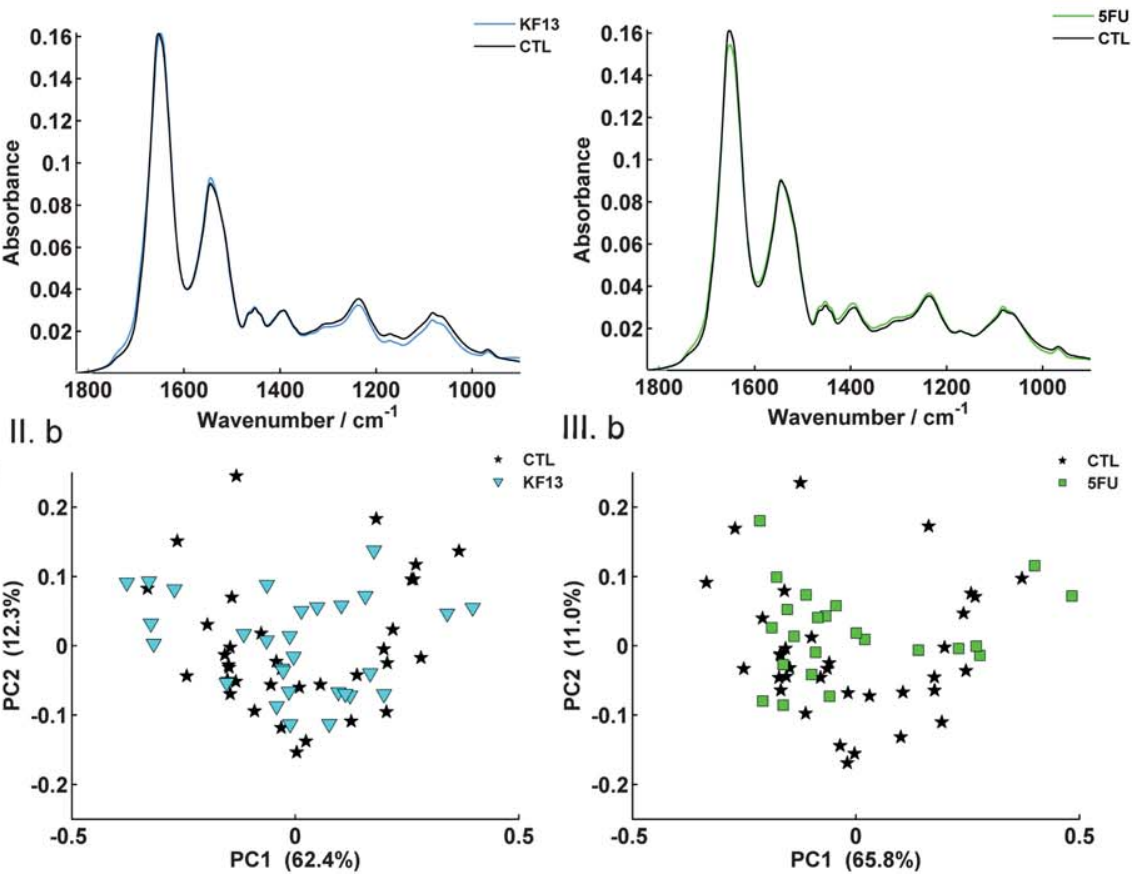

III. b

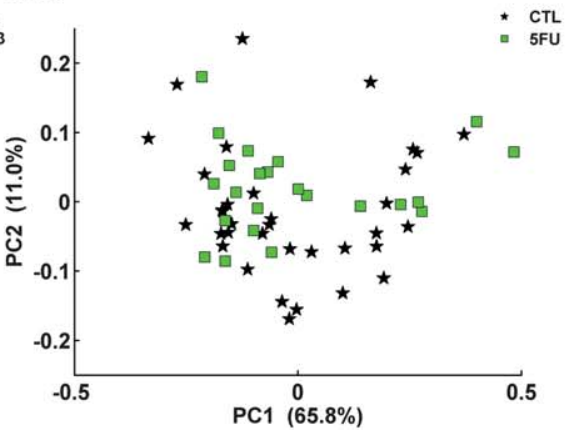

Fig. 2 (a) Mean spectra for drug treated cells versus control, (b) PCA score plots for (I) KF1, (II) KF13 and (III) 5FU respectively. 


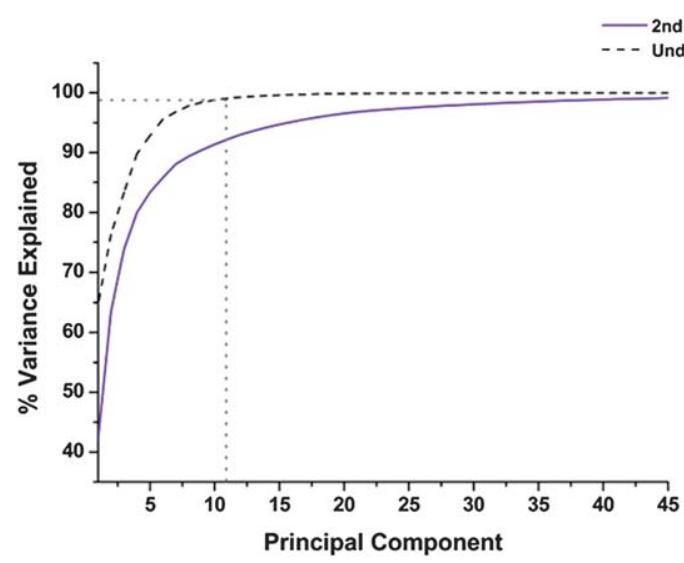

Fig. 3 Cumulative variance explained by principal components for the underivatised and equivalent $2^{\text {nd }}$ derivative dataset. There is $\sim 10 \%$ difference in VE using 12 PCs between the two groups.

there will be only one discriminant function that will be meaningful, i.e. one discriminant axis for the two groups. This indicates that these regions covering the amide bands were not informative in terms of describing different chemical signals between the control and the drug treated cells at the particular level of variance explained.

LDA was re-analysed using 14 PCs, accounting for $95 \%$ of variance in the $2^{\text {nd }}$ derivative dataset (Fig. $4 b$ and c). The LDA score plots in all cases showed only some distinction, partially separating control cell spectra from those treated with the agents (Fig. 4b). For the loadings observed for DF1 (Fig. 4c), there are two common markers that stand out that are not present in the loadings of DF2 (not pictured), suggesting that the markers are informative with regard to the group separation. The first, at $966 \mathrm{~cm}^{-1}$, is associated with $\mathrm{C}-\mathrm{O}$ stretching of the phosphate monoester group of phospholipids, phosphorylated proteins and nucleic acids. ${ }^{22}$ This peak in the loading positively correlates with $1084 \mathrm{~cm}^{-1}$ peak in KF13 and $5 F U$ and $1051 \mathrm{~cm}^{-1}$ in KF1. The assignment of $\sim 1084 \mathrm{~cm}^{-1}$ is mainly linked to a symmetric stretch $\nu_{\mathrm{s}}\left(\mathrm{PO}_{2}{ }^{-}\right)$of the phosphodiester group of nucleic acids and membrane phospholipids, and partially protein (amide III). The absorption at $\sim 1051 \mathrm{~cm}^{-1}$ is associated with $\mathrm{C}-\mathrm{O}-\mathrm{C}$ stretching of DNA and RNA. ${ }^{22}$ A second common marker from the drugtreated cell spectra (versus control) is found at $\sim 1470 \mathrm{~cm}^{-1}$, connected to $\delta \mathrm{CH}_{2}$ bending vibration of lipids and proteins and scissoring vibrations of the acyl chains in phospholipids. ${ }^{22}$ Additionally KF1 appeared to have a unique marker at $1387 \mathrm{~cm}^{-1}$ associated with $\delta\left(\mathrm{CH}_{3}\right)$ bending modes of lipids.

Data groups CTL, 5FU, KF1 and KF13 were combined and analysed as a whole dataset for $79 \%$ and $90 \%$ VE (ESI Fig. $3 \dagger$ ) resulting with poor discrimination. Fig. 5 displays the PC-LDA results for $95 \%$ variance. The variance explained for $95 \%$ in the $2^{\text {nd }}$ derivative data equated to $16 \mathrm{PCs}$. There is evidence to suggest that cells are responding most by KF13 relative to KF1 after 24 hours of exposure, as the KF13 cluster, projecting furthest from CTL in DF1 space. This was also proposed at the single-drug variable analysis (Fig. 4b). 5FU clustering was more distinctive in DF2 space. This may suggest that the cells are responding differently, or that the drug mechanism of action is different in $5 \mathrm{FU}$.
Determining the PC cut-off by monitoring increasing contributions of noise may serve as a practical way to deduce a threshold. ${ }^{23}$ Ultimately, adding more and more PCs will introduce more noise as exampled (ESI Fig. $4 \dagger$ ); whereby the first PC to introduce $99 \% \mathrm{VE}$ is 47 . The example illustrates a definitive case of over-fitting the data, as while the clustering in the DF score plot is perfect, no markers can be resolved above the nose in the loading plot analysis. For 95\% VE, however (Fig. 5a), the group cluster separation suggests some level of distinction. Spectral markers at $966 \mathrm{~cm}^{-1}$ and $1084 \mathrm{~cm}^{-1}$, also found in the single group analysis (Fig. 4) were more clearly resolved and the noise did not appear to impact upon the resolution of these specific signals.

Differences between spectral drug-treatment groups were also apparent when mean $2^{\text {nd }}$ derivative peak minima were qualitatively assessed at (1) $1171 \mathrm{~cm}^{-1}$; (2) $1155 \mathrm{~cm}^{-1}$; (3) $1084 \mathrm{~cm}^{-1}$; (4) 1061; (5) $968 \mathrm{~cm}^{-1}$ (ESI Fig. 5†). These spectral regions are said to be associated with (1) $\nu_{\text {as }}(\mathrm{CO}-\mathrm{O}-\mathrm{C})$; (2) $\nu_{\mathrm{s}}(\mathrm{C}-\mathrm{O})$; (3) $\nu_{\mathrm{s}}$ $\left(\mathrm{PO}_{2}{ }^{-}\right)$; (4) $\nu_{\mathrm{s}}(\mathrm{C}-\mathrm{O})$ and $(5) \nu_{\mathrm{s}}(\mathrm{C}-\mathrm{O})$. Markers (3) and (4) correlate with the known 'triad of nucleic infrared peaks' which are characteristic of nucleic acid vibrations at $1031 \mathrm{~cm}^{-1}$, $1060 \mathrm{~cm}^{-1}$, and $1081 \mathrm{~cm}^{-1} \cdot{ }^{24}$ Specifically, marker (3) is associated with the phosphodiester bonds of DNA and (4) is due to stretching $\mathrm{CO}$ of deoxyribose.

\section{Single point spectra (mean cell population spectra)}

SR-FTIR single cell analysis has highlighted issues affecting interpretation where it is difficult to distinguish different drugtreatment groups in PCA space. ${ }^{16}$ One explanation may be due to a high level of data complexity, resulting from parallel processes occurring from within the cell. Although some distinction can be achieved in PC-LDA, the issue of choosing the number of PCs for LDA is also concerning. Upon consideration of the current issues associated with SR-FTIR single-point spectra, the benchtop approach was employed. The advantage of this method being that a large aperture can be used to capture the average signal of several cells in one sample area. It has been previously noted in a similar experiment that a benchtop single point approach with an open aperture, sampling many cells simultaneously, can provide spectra that separate in PCA where a single cell approach on the same dataset cannot. ${ }^{16}$ It may be possible that an average signal from a cell population will reduce the impact of the cell-cycle signatures, as most cells fall into $G_{1}$ phase under normal conditions therefore the mean signal from groups of cells will be predominantly a $G_{1}$ phase profile, reducing the effects of cell-cell signatures and increasing the signals of the cellular response to the drugs.

Longer time points of drug exposure (towards 144 hours of exposure) were examined to explore the possibility of detecting changing cellular responses across a longer time period. KF13treated cells were considered for 3 and 6 days respectively and 5FU-treated cells for 6 days after initial drug exposure. For validation, cell viability was assessed using the trypan blue exclusion assay over the 6 day period.

KF13-treated cell spectra were distinguishable against the control at 3 days of exposure and was achievable in PCA and without the use of derivates or LDA. This adds weight to the suggestion cell heterogeneity masks the affects of drug treatment 
I. a

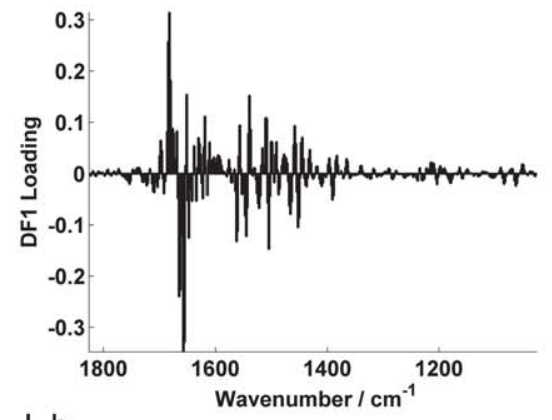

I. b

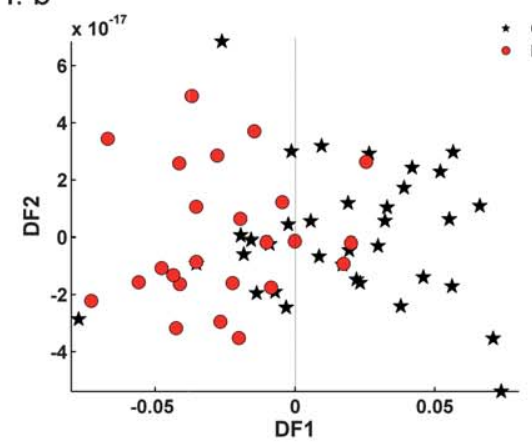

I. C

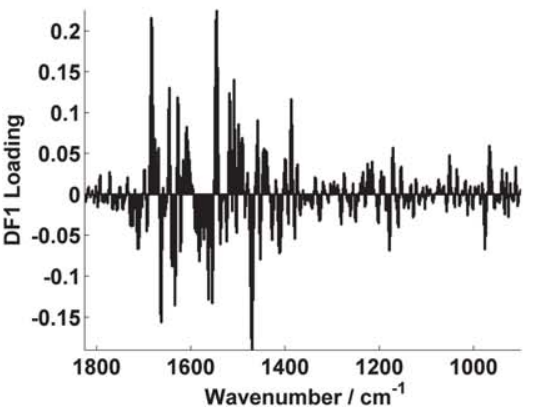

II. a

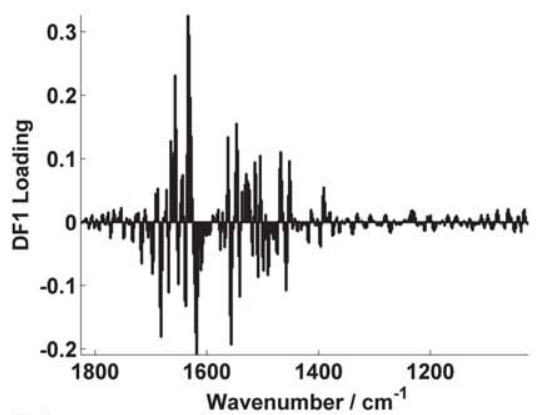

II. b

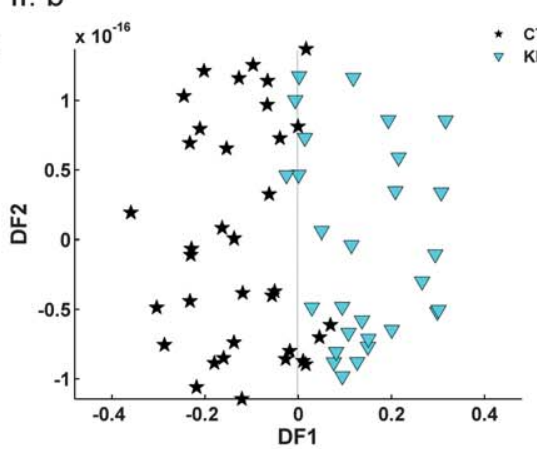

II. C

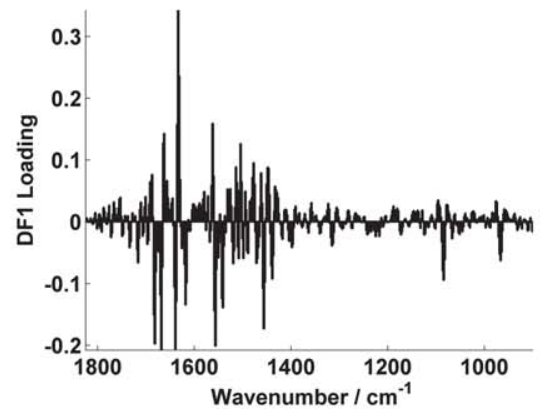

III. a

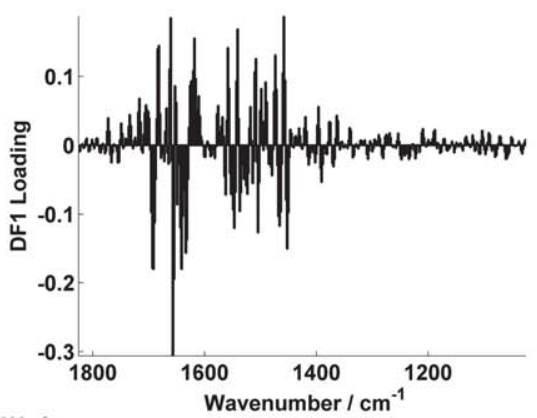

III. b

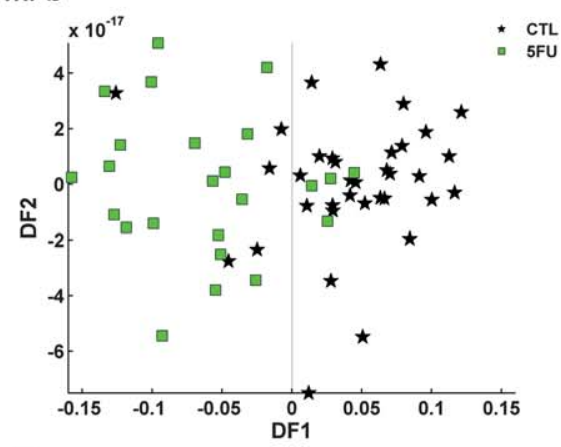

III. C

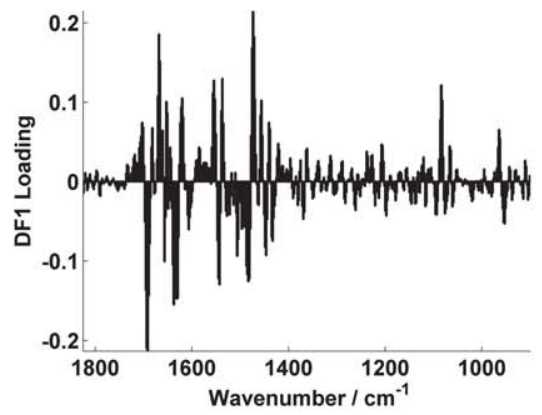

Fig. 4 KF1 (I), KF13 (II) and 5FU (III) displaying LDA loadings (a) using 90\% dataset variance from PCA (b) using 95\% variance scores and subsequent LDA loadings in DF1 for the $2^{\text {nd }}$ derivative dataset.
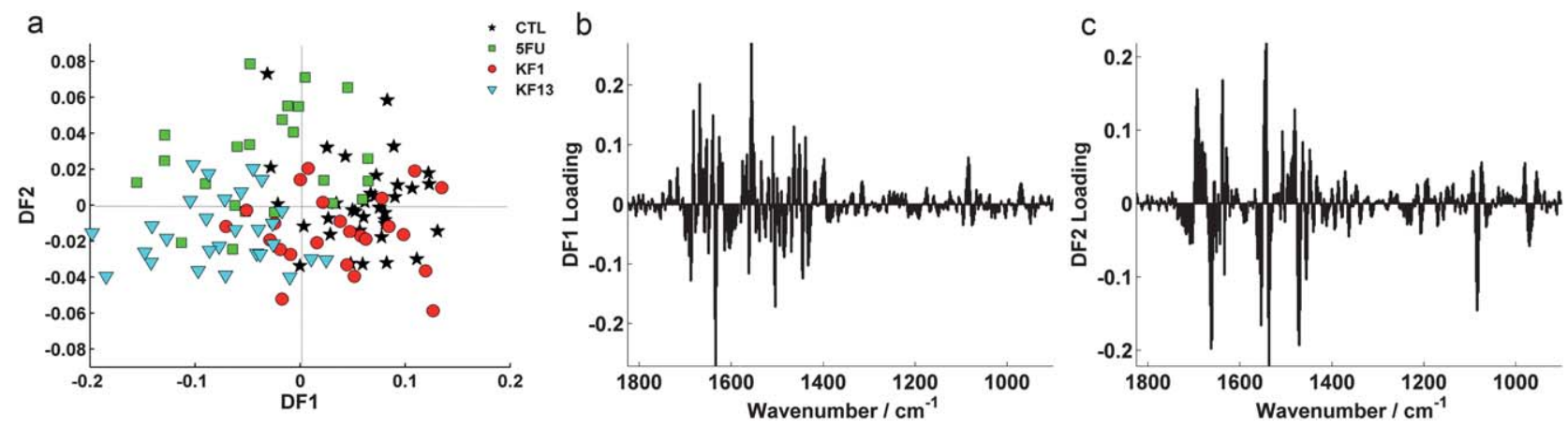

Fig. 5 LDA score plot (a) and loadings (b and c) for $2^{\text {nd }}$ derivative of the total group dataset using 16 PCs (95\% VE).

in single cell analysis. The results demonstrate that KF13 shows signs of some activity, being able to illicit some response in the cell (Fig. 6a). No discrimination between KF13 and CTL data was found in PC1 (not pictured) after 3 days of exposure and hence the loading may reflect cell cycle differences (Fig. 6c), however discrimination in PC2 reflects the difference in cell spectra between drug treated cells (KF13) and CTL cells after 3 days. There are slight differences in nucleic acids but the dominant differences are characterised by protein spectral regions and the $\mathrm{C}=\mathrm{O}$ stretching lipid-ester band at $\sim 1760-1780 \mathrm{~cm}^{-1}$. After 
a

c

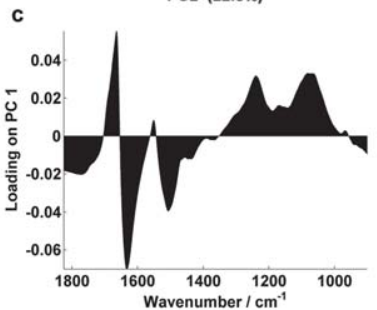

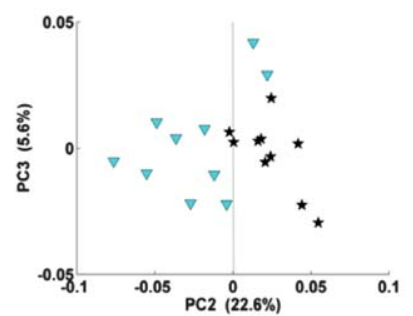

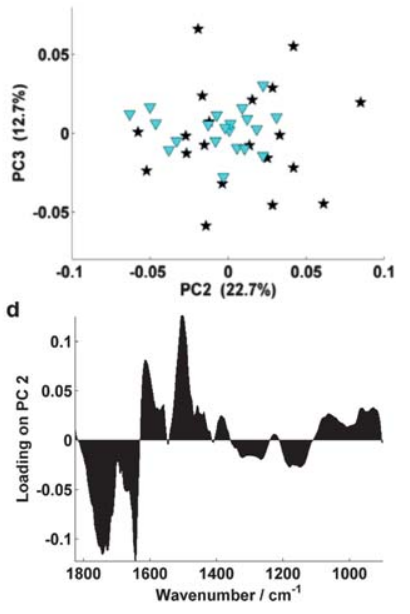

Fig. 6 PCA of average cell population spectra of (a) KF13 versus CTL after 3 days (b) KF13 versus CTL after 6 days (c) PC1 displayed no discrimination for KF13 after 3 days and hence the loading may reflect cell cycle differences (d) chemometric discrimination in PC2 reflects the difference between KF13 and CTL cells after 3 days.

six days from KF13 drug exposure (Fig. 6b), however the drugtreated cell spectra are indistinguishable from the control, suggesting that the cells may have recovered.

Visually, the physical difference in condition of 5FU-treated cells against the CTL/KF13 cells is very apparent after six days of exposure. The morphological differences are illustrated in the in vitro photomicrographs taken for CTL, 5FU and KF13 in Fig. 7(ac). The 5FU-treated cells (Fig. 7b) have a swollen appearance, with instances of cell blebbing in contrast to the CTL and KF13 images (Fig. 7a and c). In contrast, KF13 treated cells appeared similar in morphology to the control cells, suggesting a similar viability. This was confirmed by a count of viable cell confluence over a 6 day period (Fig. 7d). The remaining viable 5FU-exposed cells no longer appeared to proliferate; their cytostatic phenotype suggesting more permanent DNA damage. The KF13-exposed cells, however, appeared to recover from proliferation inhibition after three days. The assay gave a valid explanation for the discrimination of 5FUcell spectra from the chemistry of the CTL/KF13-treated cell spectra in the PCA score plot (Fig. 7e).

After 6 days from initial drug exposure, KF13 cell spectra were undistinguishable from control cell spectra, suggesting cellular recovery. A possible explanation is that the drugs were steadily effluxed out of the cells, i.e. the drug effect was no longer observed as the drug was no longer present; P-gp and other membrane transporters such as MRP1, and ABCG2 recognize a wide spectrum of compounds, however, they share a common feature in that their transport substrates are typically hydrophobic, especially the case for P-gp and ABCG2. Therefore many drugs that exhibit similar hydrophobic properties could be substrates for these drug efflux pumps and may readily cross lipid bilayer by passive diffusion. ${ }^{25}$ For P-gp substrates, the now widely accepted 'hydrophobic vacuum cleaner' model, accounts for the lipophilic nature of P-gp substrates. ${ }^{25-27}$ It is suggested that drugs that have entered into the phospholipid bilayer are pumped out of the membrane by the P-gp pump and are expelled into the extracellular aqueous phase.

Drug efflux could be analysed further by blocking the efflux ability of the cell population in a time course fluorescence-activated cell sorting (FACS) experiment. Verapamil, for example, blocks the efflux ability of an entire cell population and has been previously used to provide a FACS gate for sorting of 'side population' cells. These cells are enriched for stem-like cells from a 'normal population of cells. ${ }^{28}$

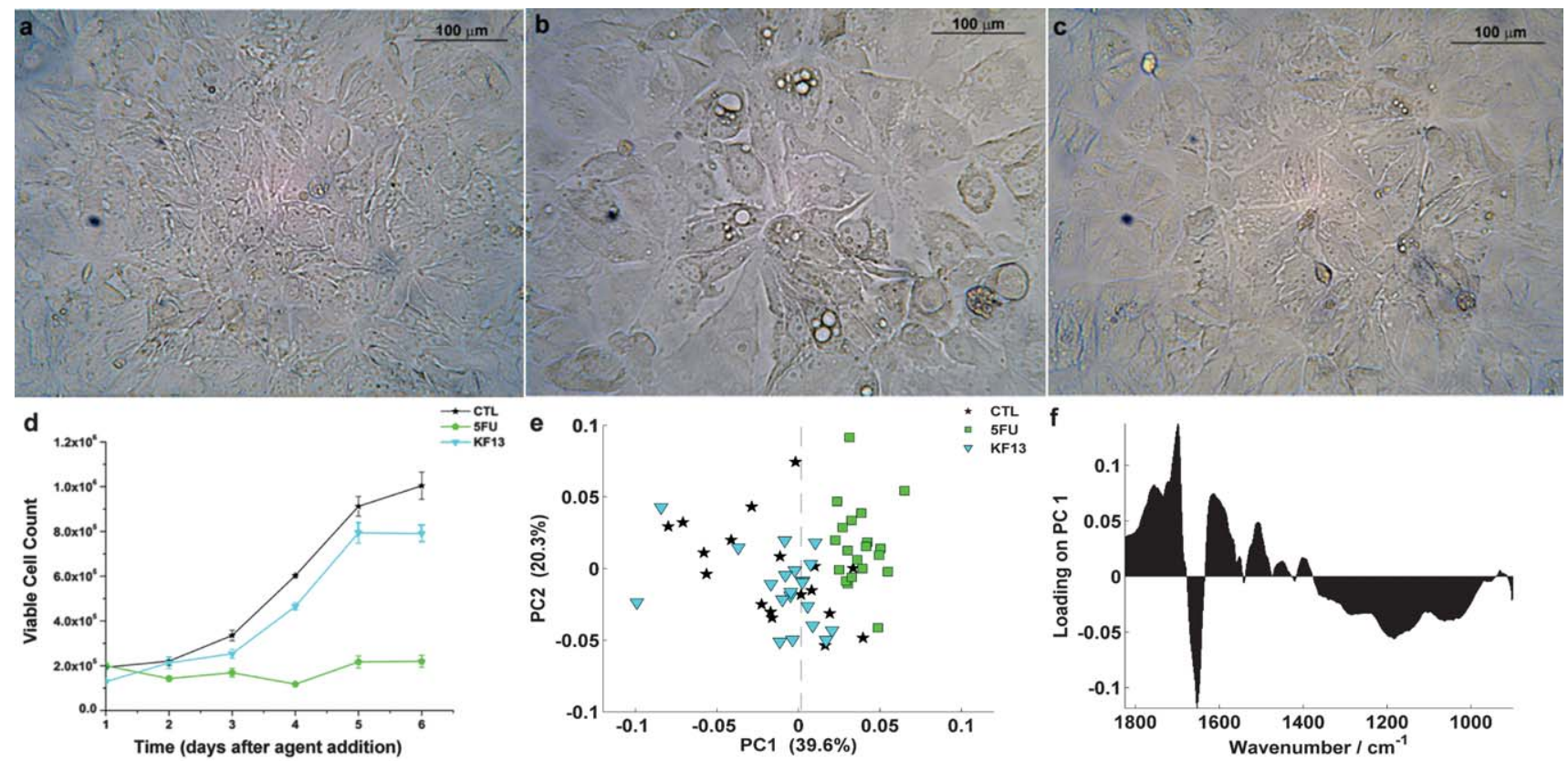

Fig. 7 In vitro photo-micrographs of the Caki-2 cells after 6 days of drug exposure to initially sub-confluent cells: (a) CTL, (b) 5FU and (c) KF13. (d) Cell viability proliferation assay for CTL, KF13 and 5FU treated cells; (e) PCA score plot of PC1 versus PC2 for 5FU and KF13 versus CTL after 6 days of exposure and (f) respective loading plot for PC1. 
The loading plot for PC1 highlights anti-correlated differences in chemistry between the 5FU-treated cell spectra and the CTL/ KF13 predominantly for nucleic-acid related regions where the drugs are thought to target, but also changes within the amide I around $\sim 1650 \mathrm{~cm}^{-1}$, suggesting changes in protein secondarystructural conformation (Fig. 7f).

\section{Conclusions}

In this case, the Caki-2 cell line was not particularly sensitive to the experimental gold analogues, however other cell lines will be tested for efficacy. In contrast, Caki-2 was clearly sensitive to 5FU, which acted as a reliable control to deduce cellular signatures.

Overall, the combination of the spectral interpretation and use of complementary biological assays for validation provides some stimulating results that seem to be in agreement. Analysing this difference by capturing mean single point spectra across a population alone, however, may be misleading. Theoretically cells are randomly distributed and enough regions of the sample are analysed so that the mean result should be representative of that sample population. In reality, however, this representative signal is biased. In a typical cell population, for example, there are more cells in $G_{1}$ phase than any other phase, therefore a mean signal from the population is more of a reflection of the cell signals in $\mathrm{G}_{1}$. This highlights the need for single cell spectral acquisition.

The results of the PC-LDA displayed some promising cellular response markers in a non-ideal situation; The use of derivates inherently reduces signal to noise and the extended use of principal components ultimately leads to a lack in confidence in correct interpretation of the results due to over-fitting. In order for single cell analysis to be more effective, however, there is a clear requirement for further methodological investigation towards a simplification step. Differences in cell cycle chemistry can be separated from the evaluation of spectral cellular response signatures to different therapeutic compounds. Clearly, PCA alone can achieve the desired result if intra-cellular heterogeneity can be separated from consideration in the analysis of drug-cell interactions.

\section{Acknowledgements}

We acknowledge the EPSRC-RSC Analytical Science Studentship scheme and also the University of Manchester for financial and technical support for $\mathrm{CH}$. We acknowledge SOLEIL for provision of synchrotron radiation facilities and we would like to thank the EU for funding travel under the ELISA programme. We also thank Paul Dumas and all staff at SOLEIL associated with the SMIS beamline.

\section{References}

1 A. Lamberti, C. Sanges and P. Arcari, Spectroscopy, 2010, 24(5), 535546.

2 G. A. R. Ahmed, F. A. R. Khorshid and T. A. Kumosani, Int. J. Nano Biomater., 2009, 2(1-5), 396-408.

3 J. R. Mourant, Y. R. Yamada, S. Carpenter, L. R. Dominique and J. P. Freyer, Biophys. J., 2003, 85(3), 1938-1947.

4 R. Zhao, L. Quaroni and A. G. Casson, Analyst, 2010, 135(1), 53-61.

5 L. Di Giambattista, D. Pozzi, P. Grimaldi, S. Gaudenzi, S. Morrone and A. Congiu Castellano, Anal. Bioanal. Chem., 2011, 1-8.

6 U. Zelig, J. Kapelushnik, R. Moreh, S. Mordechai and I. Nathan, Biophys. J., 2009, 97(7), 2107-2114.

7 C. Petibois and G. Deleris, Cell Biol. Int., 2005, 29(8), 709-716.

8 R. H. Shoemaker, Nat. Rev. Cancer, 2006, 6(10), 813-823.

9 K. Flower and A. McGown, US Pat., 7732485 B2, 2010.

10 R. H. Shoemaker, Nat. Rev. Cancer, 2006, 6, 813-823.

11 V. Vichai and K. Kirtikara, Nat. Protoc., 2006, 1, 1112-1116.

12 E. Gazi, J. Dwyer, J. Miyan, P. Gardner, C. Hart, M. Brown and N. W. Clarke, Biopolymers, 2005, 77, 18-30.

13 P. Dumas, F. Polack, B. Lagarde, O. Chubar, J. L. Giorgetta and S. Lefrancois, Infrared Phys. Technol., 2006, 49(1-2), 152-160.

14 P. Bassan, H. J. Byrne, F. Bonnier, J. Lee, P. Dumas and P. Gardner, Analyst, 2009, 134(8), 1586-1593.

15 P. Bassan, A. Kohler, H. Martens, J. Lee, H. J. Byrne, P. Dumas, E. Gazi, M. Brown, N. Clarke and P. Gardner, Analyst, 2010, 135(2), 268-277.

16 K. R. Flower, I. Khalifa, P. Bassan, D. Demoulin, E. Jackson, N. P. Lockyer, A. T. McGown, P. Miles, L. Vaccari and P. Gardner, Analyst, 2011, 136(3), 498-507.

17 M. Defernez and E. K. Kemsley, Trends Anal. Chem., 1997, 16, 216221.

18 H. F. Kaiser and W. T. Norman, Psychol. Rep., 1991, 69(1), 111-114.

19 G. Y. Kanyongo, J. Mod. Appl. Stat. Meth., 2006, 5(2), 332-343.

20 M. W. Watkins, State College, PA: Ed \& Psych Associates, 2000.

21 R. D. Ledesma and P. Valero-Mora, Practical Assess. Res. Eval., $2007,12,1-11$.

22 Z. Movasaghi, S. Rehman and I. U. Rehman, Appl. Spectrosc. Rev., 2008, 43(2), 134-179.

23 M. C. M. Grimbergen, C. F. P. Van Swol, C. Kendall, R. M. Verdaasdonk, N. Stone and J. L. H. R. Bosch, Appl. Spectrosc., 2010, 64(1), 8-14.

24 L. Chiriboga, P. Xie, H. Yee, V. Vigorita, D. Zarou, D. Zakim and M. Diem, Biospectroscopy, 1998, 4(1), 47-53.

25 P. D. W. Eckford and F. J. Sharom, Chem. Rev., 2009, 109(7), 29893011.

26 C. F. Higgins and M. M. Gottesman, Trends Biochem. Sci., 1992, 17(1), 18-21.

27 R. G. Del Moral, A. Olmo, M. Aguilar and F. O'Vaue, Exp. Nephrol., 1998, 6(2), 89-97.

28 C. Hughes, M. Liew, A. Sachdeva, P. Bassan, P. Dumas, C. A. Hart, M. D. Brown, N. W. Clarke and P. Gardner, Analyst, 2010, 135, 3133-3141. 